\title{
ONLINE- BZW. DISTANZLERNEN IN DEN USBEKISCHEN HOCHSCHULEN - PROBLEME UND HERAUSFORDERUNGEN
}

\section{Islomov Sanjar Ruzikulovich}

Lehrstuhlinhaber für Deutsche Sprache und Literatur an der Staatlichen Universität Termes, Oberlektor

Termes, Usbekistan

\author{
ЎЗБЕКИСТОН ОЛИЙ ТАЪЛИМ МУАССАСАЛАРИДА ОНЛАЙН ВА \\ ДИСТАНЦИОН ТАЪЛИМ - МУАММОЛАР ВА ЕЧИЛИШИ \\ ЛОЗИМ БЎ ЛГАН ЧАҚИРИҚЛАР \\ Санжар Рўзиқулович ИСЛОМОВ \\ Немис тили ва адабиёти кафедраси мудири, катта ўқитувчи \\ Термиз давлат университети \\ Термиз, Ўзбекистон
}

\author{
ONLINE AND DISTANCE LEARNING IN UZBEK HIGHER EDUCATIONAL \\ INSTITUTIONS - PROBLEMS AND CHALLENGES

\section{Sanjar Ruzikulovich ISLOMOV} \\ Senior Teacher \\ Head of the Department of German Language and Literature \\ Termez State University \\ Termez, Uzbekistan islomov@tersu.uz s islomov@yahoo.de
}

\section{UDC (УЎК, УДК): 372.881 .1}

For citation (иқтибос келтириш учун, для цитирования):

Islomov S. R. Online-Bzw. Distanzlernen in den Usbekischen Hochschulen - Probleme und Herausforderungen // Ўзбекистонда хорижий тиллар. — 2021. — № 2 (37). — Б. $12-25$

https://doi.org/10.36078/1620221000

Received: February 12, 2021

Accepted: April 17, 2021

Published: April 20, 2021

Copyright (c) 2021 by author(s) and Scientific Research Publishing Inc.

This work is licensed under the Creative Commons Attribution International License (CC BY 4.0).

http://creativecommons.org/licenses/by/4.0/

\section{Open Access}

Abstract. Der Artikel befasst sich mit den Problemen der Onlinebzw. Distanzlernens in der Hochschulbildung. Zunächst wird der Begriff Distanzlernen geklärt. Anschließend werden auf die rechtlichen Grundlagen für das Distanzlernen in usbekischen Bildungseinrichtungen eingegangen. Danach werden zunächst die Probleme und Herausforderungen des Distanzlernens bzw. OnlineLernens anhand von empirischen Beobachtungen und mündlichen Befragungen unter Lehrenden und Lernenden gesammelt. Dabei wurden einschlägige Probleme sowohl aus der Perspektive der Lehrenden, als auch aus der Perspektive von Lernenden berücksichtigt. Anhand der gesammelten Problemfragen wurde dann ein Umfragebogen entwickelt sowie unter den Studierenden der Universität Termes durchgeführt. Laut der Umfrageergebnisse lassen sich nämlich die Probleme, die im Zusammenhang mit Onlineunterricht auftreten, generell in technische, finanzielle, persönliche und methodisch-didaktische sowie lernpsychologische Gruppen einteilen. Abschließend wurden alle Problemgruppen eingehend analysiert. Insbesondere die lernpsychologische Probleme wurden sehr umfassend behandelt, weil beim Online- bzw. Distanzunterricht die Lernenden öfters über den Umfang von Lernstoff, über den Lernort, über die Lernzeit und über den Lerntempo selbst entscheiden müssen und all das vom Lernenden ein hohes Maß an Selbststeuerung und autonomes Lernen verlangt, eine Fertigkeit also, die leider in den entsprechenden Curricula als Fach noch nicht etabliert hat.

Schlüsselwörter: Distanzlernen; Online-Lernen; Präsenz-Lernen; Hochschulbildung; Fernstudium; Ad hoc Lösung; Probleme; Herausforderungen; Hochschule; Bildungseinrichtungen; Lehrenden; 
Lernenden; E-Learning; Lernplattform; Umfrage; Studierende; empirische Beobachtung; Selbststeuerung; autonomes Lernen; Fertigkeit.

Аннотация. ннотация. Мақолада олий таълимда онлайн ва масофавий ўқитиш муаммолари кўриб чиқилди. Масофавий ўқитиш атамасига муаллиф томонидан таъриф бериб ўтилди. Шунингдек, Ўзбекистон ОТМда масофавий ўқитишнинг хуқуқий-норматив асослари мухокама қилинди. Мақоланинг амалий қисмини талабалар ва ўқитувчилар ўртасида ўтказилган онлайн ва масофавий ўқитишнинг муаммоларига бағишланган эмпирик кузатувлар тавсифи ташкил этди, натижалар оғзаки сўровлар ёрдамида йиғиб чиқилди. Сўровномада онлайн таълимнинг тегишли муаммолари бўйича нафақат ўқитувчи, балки талаба перспективаси хам хисобга олинди. Йиғилган муаммолар ёрдамида аноним сўровнома ишлаб чиқилиб, Термиз давлат университети талабалари ўртасида ўтказилди. Сўровнома натижаларига кўра, онлайн ва дистанцион таълим билан боғлиқ муаммоларни техник, молиявий, шахсий, методик-дидактик хамда психологик гурухларга бўлиш мумкинлиги мақолада ўз аксини топди. Бундан ташқари, мавзу доирасида ўрганиш психологияси билан боғлиқ бўлган муаммоларга хам батафсил тўхталиб ўтилди. Якуний хулосалар сифатида онлайн ва масофавий таълим олиш жараёнида талабалар кўпинча ўкув материали хажми, онлайн ўқиш жойи, ўрганиш вақти ва ўрганиш тезлиги хақида мустақил қарор қабул қилишлари лозим бўлиши ва бунинг учун талабалардан юқори даражадаги ўз-ўзини бошқариш ва автоном ўқиш кўникмалари талаб қилиниши ва, афсуски, мазкур кўникмаларни шакллантирувчи фан кўпгина ўқув режаларига хозирча киритилмагани каби сабаблар келтирилди. Олинган натижа ва хулосалар соха доирасидаги нафақат назарий, балки амалий билимларни хам кенгайтиришга хизмат қилади.

Калит сўзлар: масофавий ўқитиш; онлайн режимида ўқитиш; юзма-юз ўрганиш; олий таълим; сиртқи таълим; вақтинчалик ечим; ўкув платформаси; эмпирик кузатув; автоном ўқиш.

Abstract. The article is devoted to the problems of online and distance learning in the context of higher education. It deals with the legal basis for distance learning in Uzbek educational institutions, the problems of distance learning and online learning found out on the basis of empirical observations and oral surveys among teachers and learners of Termez University. Relevant problems were taken into account both from the perspective of the teacher and the learners. According to the results of the survey, the problems that arise in online teaching can generally be divided into technical, financial, personal, methodological-didactic and learning psychological groups, which were analyzed in detail. In particular, the psychological problems of learning were dealt comprehensively, because in online or distance learning the learners often have to decide for themselves about the scope of learning material, the space and time for learning, that requires from the learner a high grade of self-control and autonomous learning, a skill that unfortunately has not yet been established as a subject in the relevant curricula.

Keywords: distance learning; online learning; face-to-face learning; higher education; ad hoc solutions; problems; challenges; university; educational institutions; teachers; learners; e-learning; learning platform; survey; students; empirical observation; self-control; autonomous learning; skill. 
Es ist vielen bekannt, dass die Covid-19 bedingte Lock-down unter anderem auch die Hochschulen vor neuen Herausforderungen gestellt hat. Folgende Artikel befasst sich mit der Schwierigkeiten und Herausforderungen von Online-Lernen bzw. Distanzlernen im Fremdsprachenunterricht, insbesondere im DaF-Unterricht.

Bevor auf die Thematik des Artikels eingegangen wird, soll hier der Begriff Distanzlernen geklärt werden. In der einschlägigen deutschen Fachliteratur werden hier für die Begriffe Fernstudien, Fernlernen und Distanzlernen aufgeführt, wobei alle diese Begriffe synonymisch verwendet werden. Definiert werden diese Begriffe vor allem aus räumlichen Perspektive, mit anderen Worten die räumliche Trennung von Lerner und Lehrinstitution bzw. Lehrende. Alle diese Begriffe stehen dann als Gegenpole zum Begriff Präsenzlernen, wo die Lernenden und die Lehrenden zeitgleich in einem Raum anwesend sind und der Lernprozess in der Form von „face to face“ stattfindet (Vgl. 8, 1192-1193; 6, 195). Im folgenden Artikel wird der Begriff Distanzlernen verwendet, da dieser mit dem Einzug von digitalen Medien in Lernkontexte seine Bedeutung erweitert hat und die Situation in den Bildungseinrichtungen von Usbekistan besser beschreiben kann. „Der Begriff Distanzlernen umfasst also alle Formen des Fern- und Tele-Lernens und spielt somit ebenfalls an konventionellen Universitäten, die virtuelle Seminare anbieten, oder bei Firmen, die ihren Mitarbeitern arbeitsplatznahe mediengestützte (computer- und/ oder webbasierte) Weiterbildungen anbieten, eine Rolle.“ $(8,1193)$.

Ferner ist insbesondere in der Hochschulbildung zu unterscheiden zwischen Distanzlernen und Fernstudium als Form des Studiums sowohl in usbekischen, als auch in deutschen Hochschulen. In dieser Form des Studiums werden die Lehrveranstaltungen entweder komplett ohne übliche Präsenzphasen, wo die Präsenzphasen durch E-Learning Plattformen, Apps oder Online-Lernen ersetzt werden, oder Distanzlernen mit Präsenzlernen kombiniert werden (Vgl. 4).

Mit dem Ausbruch der weltweiten Pandemie wegen der Atemwegserkrankung Covid-19 und zunehmenden Corona-Lockdown in fast allen Ländern der Welt, standen plötzlich auch in Usbekistan alle Bereiche des öffentlichen Lebens vor neuen Herausforderungen. Distanzarbeit oder Home-Office war die entsprechende eindeutige Antwort vieler Akteure des öffentlichen Lebens. Und die Begriffe wie Distanzlernen, Fernlernen, Fernunterricht, Fernstudien, Onlinelernen und Lernplattformen waren in der Bildungseinrichtungen plötzlich in aller Munde. Während für einige Lehrende diese Begriffe, sowie die Formen des Distanzlernens einigermaßen geläufig waren, war es für die große Mehrheit der Lehrenden eher unbekannt. Gerade die letzteren waren deshalb skeptisch und betrachteten alle Formen des Online-Unterrichts und Distanzlernens eher als Ad hoc Lösung.

Und die Skeptiker hatten diesbezüglich zumindest aus rechtlicher Sicht zum Teil Recht. Da in den entsprechenden einschlägigen usbekischen Gesetzen und Verordnungen keine explizite Anweisungen zum Thema Distanzlernen und Onlinelernen geäußert waren. Die rechtliche Grundlage für das Distanzlernen existierte nur in beschränktem Maße in der Hochschulbildung (Vgl. 2). Gemäß der Verordnung des Ministerkabinetts der Republik Usbekistan №930 vom 21.11.2017 „Über die Bestätigung der Studienordnungen in den Fern- und Abendstudiengängen in den Hochschulen" wurde es erlaubt, während des Studiums in den oben erwähnten Studiengängen auf die Elemente des Distanzlernens zuzugreifen. 
Mit der Annahme von neu aufgelegten Bildungsgesetz der Republik Usbekistan №637 vom 23.09.2020 wurde dann die rechtliche Grundlage für Distanzlernen für alle Einrichtungen geschaffen. Artikel 16 dieses Gesetzes besagt, dass Lernprozess gemäß der entsprechenden Curriculum in der Form des Distanzlernens stattfinden kann. Die Einzelheiten sowie in welcher Form Fernstudium organisiert wird, wird allerdings gemäß der einschlägigen Verordnung des Ministerkabinetts der Republik Usbekistan geregelt (Vgl. $1)$.

Online-bzw. Distanzlernen geben einerseits beim Fremdsprachenlernen viele Möglichkeiten. Da mit der Entwicklung der Medien sowie deren Integration ins Online-Lernen wird es ermöglicht nicht nur auf die Fertigkeiten wie Lesen und Schreiben sich zu beschränken, wie dies früher der Fall war, sondern mit Hilfe der neuen Informationstechnologien kann nun der Distanzlernen interaktiv, kommunikativ und mündlich-visuell gestaltet werden (Vgl. 8, 1194; 6, 195). Andererseits aber ist Fremdsprachenlernen an sich sehr komplizierter Vorgang, und eine Sprache-Online zu lernen macht die Sache noch schwerer.

Über die Probleme des Distanzlernens im DaF-Unterricht wurden die empirischen Beobachtungen des Autors während der monatelangen Tätigkeit als online DaF-Lehrers in der Corona-Lockdown Zeit berücksichtigt. Um aber die Problematik des Online- bzw. Distanzlernens objektiv und eingehend behandeln zu können, hat der Autor entschieden eine Umfrage unter den Studierenden durchzuführen. Dafür entwarf der Autor eine einschlägige Umfrage, die zu allen Facetten des Online-Lernens und sogar auch zur Qualität des Online-Unterrichts sowie zur Fachkompetenz der Lehrenden Fragen enthielt. Damit die Probanden bei der Beantwortung der Fragen frei und objektiv fühlen können, wurden Umfragen anonym und geheim durchgeführt [Siehe Anhang]. Zunächst werden die allgemeinen Informationen über die Umfrage und anschließend werden auf die Fragen umfassend eingegangen.

Alle Befragten waren die Studierenden der Termeser Staatlicher Universität und studierten Deutsch, Englisch und Russisch als Hauptfach. Insgesamt haben an der Umfrage 64 Personen teilgenommen, 27 davon studierten im dritten und 37 im zweiten Studienjahr. Die Studenten des 1. Studienjahres wurden gezielt nicht berücksichtigt, weil sie erst seit kurzem ihr Studium angefangen haben. 12 Studenten oder fast ein Fünftel der Befragten kommen aus anderen Provinzen, während 52 Personen oder 80 Prozent der Befragten aus der Region Surkhandarya kommen. 16 Testpersonen sind Männer, während 48 Personen Frauen sind. Das überwiegende Alter der Befragten beträgt zwischen 20 und 25 Jahren, während aber auch vereinzelt über 25 Jährige (3 Personen) und einige unter 20 Jährige (10 Personen) auch vorkommen. Interessant für die Umfrageergebnisse sind die ständigen Wohnsitze der Studierenden, da sie während der Online-Unterrichte u.a. auch wegen Corona-Lock down meistens zu Hause bei den Eltern waren. Dafür sollten die Befragten in der Umfrage angeben, ob sie in einem Bezirk- bzw. Provinzzentrum oder in einem ländlichen Ort wohnen. Laut der Umfrageergebnisse etwa ein Drittel bzw. 20 Personen der Befragten wohnen in den Bezirks bzw. Provinzzentrum, während etwa zwei Drittel oder 39 Personen in ländlichen Ort wohnen. 5 Testpersonen haben zu diesem Punkt keine Angabe gemacht.

Auf die Frage Wie bewerten Sie generell Online/Distanzlernen haben die Studierende überwiegend mit befriedigend bis sehr schlecht geantwortet. Etwa ein Fünftel der Befragten beantworteten diese Frage mit gut bis sehr 
gut. Auf die Frage Ich nahm in Online/Distanzlernen vollständig teil antworteten die Befragten folgendermaßen. Von Testpersonen, die im Bezirk/Provinzzentrum wohnen, haben mehr als die Hälfte mit gut bis sehr gut geantwortet (13 Personen) und etwa ein Drittel mit befriedigend bis sehr schlecht (7 Personen) geantwortet. Und knapp die Hälfte der Befragten (48\%), die in dem ländlichen Ort ihren ständigen Wohnsitz haben, haben in den Online-Veranstaltungen meistens teilgenommen, während die knappe Mehrheit (52\%) dieser Gruppe in Unterrichten von manchmal bis nie teilgenommen haben.

Nächste Frage in der Fragebogen war über die Ursachen des Fehlens der Teilnahme von Studierenden in den Online-Unterrichten. Falls nein, aus welchem Grund konnten Sie in Online/Distanzlernen nicht teilnehmen (mehrere Antworten möglich) lautete die Frage mit folgenden Antwortmöglichkeiten Internetzugang (Geschwindigkeit), Handyproblem, Finanzielle Probleme, Motivation/Interesse/Wille, Fehlen von PC, Qualität des Online/Distanzunterrichts, Zeitmangel wegen Hausangelegenheiten, Fehlende Kontrolle der Anwesenheit und Hausaufgaben und Kenne mich im Internet nicht gut aus. Die meistgenannte Antwort auf diese Frage war mit Abstand die Geschwindigkeit von Internet. Genau 49-mal wurde diese Antwort von den Befragten gewählt, gefolgt von Antworten Zeitmangel wegen Hausangelegenheiten sowie Fehlen von Internetkenntnissen mit jeweils 15 Nennungen. Interessant ist hier die Antwort Zeitmangel, da im Falle eines Präsenz-Unterrichts dieses Problem gar nicht zustande gekommen wäre, da die meisten Studierenden fern von Zuhause in den Studentenwohnheimen und Wohngemeinschaften wohnen würden. In den nächsten Stellen kommen mit jeweils 11-mal und 10-mal Nennungen Fehlen von eigenen Computern sowie finanzielle Probleme. Mit finanziellen Problemen sind hier die Kosten, die wegen des Internetzugangs entstehen, gemeint. Als nächste kommt der Punkt der Qualität von OnlineDistanzunterrichts mit 8 Nennungen. Interessant ist diese Frage auch deshalb, weil diese Frage in der Umfrage oben auch vorkam, wo die Teilnehmenden die Qualität der Online-Unterrichte generell bewertet hatten und fast 80 Prozent der Befragten diese Frage mit befriedigend bis sehr schlecht beantworteten und als Ursache für das Fehlen im Online-Unterricht nur 8 Personen diese Antwort gewählt hatten. Weiter kommt das Problem des Fehlens von eigenen bzw. internettauglichen Handy mit 5 Nennungen. Fehlende Kontrolle der Anwesenheit und Hausaufgaben sowie fehlende Motivation wurden jeweils 3-mal genannt.

Laut der Umfrageergebnisse lassen sich die Probleme, die im Zusammenhang mit Online-Deutschunterricht auftreten, generell in folgende Gruppen einteilen: technische, finanzielle, persönliche und methodisch-didaktische. Und damit werden auf die oben angeführte Informationen einzeln und detailliert eingegangen.

Technische Probleme. Zunächst einmal ist das Problem mit dem Internetzugang und mit der Qualität der Internetverbindung zu nennen. Es soll bemerkt werden, dass in der Provinz Surchandarja das Festnetzinternet meistens in den Städten und Bezirks- sowie Provinzzentren vorhanden sind und die große Mehrheit der Studierenden, sowie der Rest der Bevölkerung haben Zugang zum Internet ausschließlich über das Mobilfunkanbieter über ihre Handys. Besonders in abgelegten Regionen war die Situation mit Internetzugang sehr problematisch. Diese Informationen wurden auch mit Umfrageergebnissen ebenso bestätigt. Unter dieser Gruppe der Fragen befanden drei Fragen, und zwar:

oIch habe Zugang zum Internet via mein mobil Handy 
oIch habe Festnetz/ADSL-Anschluss zum Internet

oMeine Internetgeschwindigkeit ist sehr gut $(\mathrm{h}+/ 4 \mathrm{~g} / \mathrm{LTE})$

Nach der Analyse der Umfrageergebnisse lassen sich folgende Tendenzen zu entnehmen. Alle 64 Befragten können in drei Gruppen geteilt werden: Erstens die Studierenden, mit sowohl Festnetz, als auch Mobilfunk Internetzugang, zweitens die Studierenden mit nur Mobilfunk Internetzugang und drittens Studierenden, die weder Festnetz, noch Mobilfunk Internetzugang haben. Zur ersten Gruppe gehören 16 Personen, die überwiegend in der Bezirks- bzw. Provinzzentren wohnen. Von diesen 16 Testpersonen gaben 7 an, dass sie mindestens gut bis sehr gute Internetgeschwindigkeit haben, während die anderen Befragten die Geschwindigkeit mit befriedigend angaben. Bei den letzteren liegt das Problem offensichtlich bei Interanbietern oder dass sie kein Glasfaserkabel Anschluss besitzen. Zur zweiten Gruppe zählen die meisten Studierenden. 39 Studenten oder etwa $61 \%$ der Befragten haben Zugang zum Internet via ihre Handys. Die meisten studierenden dieser Gruppe wohnen in ländlichen Orten oder in Bezirkszentren. Einige Studierenden wohnen aber auch in Provinzzentren, die allerdings zu Hause kein Computer und damit auch kein Festnetzanschluss zum Internet haben. Etwa 14 Prozent oder 9 Personen haben nicht einmal Mobilfunk, geschweige denn vom Festnetz Internetzugang. Alle Befragten dieser Gruppe wohnen in ländlichen Orten bzw. in sehr weit abgelegenen Siedlungen, wo es Mobilfunk nur zum Telefonieren gibt, oder auch überhaupt kein Mobilfunknetz vorhanden ist.

Auf die Frage über die Internetgeschwindigkeit werden die Antworten noch dramatischer. Nur 10 der Befragten gaben an, dass sie überhaupt kein Problem mit der Geschwindigkeit von Internet haben, während die große Mehrheit der Befragten (32 Personen) eine ausreichende Internetgeschwindigkeit haben. 22 Befragten oder 34 Prozent haben ihren Internetanschluss von schlecht bis sehr schlecht eingeschätzt.

Wenn der Anteil der Befragten aus anderen Provinzen in dieser Umfrage in Betracht gezogen wird (12 Personen oder 19 Prozent), ist das Problem mit der Geschwindigkeit von Internet nicht nur regional, sondern als überregional einzuschätzen.

Finanzielle Probleme. Ein großes Problem hier ist, dass viele Studierende im Online-Unterricht nicht per PC oder Laptop, sondern mit Hilfe ihrer Handys teilnehmen, weil sie kein PC, geschweige denn ein Drucker haben. Denn die konstitutiven Elemente des Online-Unterrichts fordern vorproduzierte Fernkurs sowie ausgedruckte Kursmaterial (Vgl. 6, 195-196). Es kann durchaus sein, dass die Studierenden bei gutem Internetempfang, immer noch kein Zugang zum Internet haben, weil sie ganz banal kein Geld für Handy bzw. Internettarife haben oder sie ihre monatlichen Internetpakete schon aufgebraucht haben und keinen zusätzlichen Internetpaket kaufen können. Ein anderes Problem hier ist, dass die Studierende kein internetfähiges Handy oder in der Familie nur ein Handy für alle Familienmitglieder haben. Diese Informationen wurden auch mit der Umfrageergebnissen ebenso bestätigt. In diesem Teil der Umfrage war insgesamt 5 Fragen:

oInternetpakete sind teuer

oIch habe einen modernen PC

oIch habe ein Drucker zum Drucken von Materialien

oIch habe modernes, eigenes Handy

oIch habe für das Online-Unterricht Handy von meinen Eltern benutzt 
Auf die erste Frage haben die überwiegende Mehrheit der Probanden mit richtig bzw. sehr richtig beantwortet. Auf die Frage ob sie ein modernes Computer besitzen, haben 12 Befragten mit ja beantwortet. Weitere 6 Personen haben angegeben, dass sie zwar ein PC haben, aber ihre Computer nicht so modern sind. Das macht zusammen dann 28 Prozent der Befragten. Weitere 9 Personen haben zu diesem Punkt keine Antwort gegeben. 37 Studierende gaben an, dass sie kein PC besitzen. Etwa 10 Prozent der Befragten verfügen ein Drucker zu Hause. 9 Personen gaben an, dass sie kein Handy überhaupt besitzen. Weitere 8 Personen haben diese Frage offen gelassen. 15 Personen gaben an, dass sie zwar ein eigenes Handy haben, allerdings ihr Handy nicht so modern ist. Alle anderen Befragten haben ein modernes Handy. 17 Probanden oder 26\% gaben an, dass sie in OnlineUnterrichten das Handy ihrer Eltern oder ihren Familienmitglieder verwendet zu haben. Das heißt, dass auch diejenige Studierende, die ein modernes Handy besitzen, von dieser Möglichkeit Gebrauch zu machen, vermutlich aus finanziellen Gründen, da sie alleine kein bzw. beschränkten Zugang zum Internet hatten.

Persönliche Probleme. Hier kann durchaus sein, dass die Lernenden für das Fach, was sie an der Universität studieren, einfach kein Interesse haben und ihnen einfach Motivation fehlt im Unterricht teilzunehmen. Das liegt auch an der begrenzten und komplizierten Hochschulzugangsprozess für die Schulabsolventen in Usbekistan zusammen, wo Studierende ihr Studium de facto sehr schwer wechseln können, geschweige denn vom Neuanfang eines anderen Studiums. Ein weiteres Problem ist, dass die Lernenden die komplizierte Lage in der Corona-Zeit für ihre persönliche Interessen ausnutzen und die Online-Unterrichte einfach schwänzen, wohl wissend, dass ihnen nichts passieren wird und sie ihr Studium weiter fortsetzen können ohne exmatrikuliert $\mathrm{zu}$ werden oder ohne weitere Konsequenzen fürchten zu müssen. Nun wird auf die Umfrageergebnisse eingegangen. In diesem Abteil der Umfrage waren insgesamt 6 Fragen zu beantworten.

oIch habe an Online-Unterrichten in allen Fächern teilgenommen oIch habe an Online-Unterricht NUR in Hauptfächern teilgenommen oHabe an Online-Unterrichten NUR in Nebenfächern teilgenommen oDie Teilnahme an Online-Unterrichten wurde streng kontrolliert oOnline-Unterrichte hatten keinen direkten Bezug zu Note oWegen der Pandemie gaben die Lehrer die Noten einfach so Auf die erste Frage haben 17 Personen angegeben, dass sie in OnlineUnterrichten nicht teilgenommen haben. Weitere 20 Personen haben angegeben, dass sie manchmal an den Unterrichten teilgenommen haben, während andere in Distanz-Unterrichten regelmäßig teilnahmen. Was die zweite und dritte Fragen angehen, so haben 26 Prozent der Testpersonen keine eindeutige Antwort gegeben, während 8 Prozent der Teilnehmer lieber in Nebenfächern im Unterricht waren, als in den Hauptfächern. 19 Studierende gaben an, dass sie sowohl in Haupt, als auch in Nebenfächern gleichermaßen teilgenommen haben. Die große Mehrheit oder $44 \%$ der Befragten haben bevorzugt in Hauptfächern teilgenommen, als in den Nebenfächern. Auf die Frage der strengen Anwesenheitskontrolle haben die Studierende je Hälfte mit ja und je Hälfte mit nein geantwortet. Die nächste Frage lautete ob die Teilnahme an Online-Unterrichten keinen direkten Bezug zu Noten hatte. 20 Befragten oder 33\% haben hier eindeutig positioniert und gaben an, dass diese Aussage falsch ist. Weitere 22 Personen gaben an, dass diese Aussage stimmt, während andere 20 Befragten hier eine neutrale Antwort gegeben haben. Der letzten Aussage, dass die Lehrenden 
den Lernenden die Noten einfach geschenkt haben, stimmten 15 Testpersonen zu. Weitere 8 Personen konnten diese Aussage weder bestreiten noch bestätigen. Etwa 8 Prozent der Befragten haben hier keine Angaben gemacht und die große Mehrheit oder 36 Personen oder 56\% der Befragten stimmten dieser Aussage nicht zu.

Methodisch-didaktische Probleme. Didaktische Probleme sind auf den DaF-Lehrer und auf den Kursmaterial zurückzuführen. Es ist durchaus möglich, dass der Lehrer sein Kursmaterial didaktisch nicht vorbereitet hat aus welchem Grund auch das mag sein. Denn in allen Phasen des Distanzlernens ist eine gründliche Vor- und Nachbereitung von Nöten. Ferner verlangt das Distanzlernen von Studierenden an hohes Maß an autonomes Lernen sowie Selbststeuertes Lernen. Und der Lehrer soll hier sehr gut ausgebildet sein, damit er seinen Lernenden die nötigen Strategien an die Hand geben kann(Vgl. 8, 1197). Mehr dazu erfahren Sie unten in Lernpsychologischen Problemen. Ein weiterer Punkt ist der Faktor Lehrer. Es gibt einige Lehrer, die der neuen Informationstechnologien schlicht und einfach nicht gewachsen und mit dem Online-Unterricht überfordert sind. $\mathrm{Zu}$ allen oben genannten Punkten wurden in der Umfragebogen entsprechende Fragen entwickelt. Insgesamt sind in diesem Teil der Umfrage 10 Fragen enthalten:

oOnline-Lehrmaterialien (Lehrbuch, Materialien, Handouts) sind in guter Qualität

oQualität der Online-Unterrichte sind sehr gut

oDie Aufgaben für Online-Unterricht sind zu viel

oDie Aufgaben für Online-Unterricht sind zu wenig

oIch konnte bei der Erfüllung der Hausaufgaben für Online-

oUnterricht meine Zeit richtig und effektiv einteilen

oIch weiß was Autonomes/Selbstständiges Lernen ist

oIch konnte Aufgaben autonom/selbstständig machen

oDer Lehrer hat die Online-Unterrichte gut unterrichtet

oDer Lehrer ist in modernen Informationstechnologien kompetent mit PC

oDer Lehrer unterrichtete den Online-Unterricht $\square$ mit Handy $\square$

Die ersten vier Fragen beziehen sich auf die Vor- und Nachbereitung des Online-Unterrichts. Die erste Frage in diesem Teil haben 19 Studierende mit schlecht bis sehr schlecht beantwortet, während die große Mehrheit sind der Meinung, dass die Materialien in den Lernplatformen ausreichend und in guter Qualität waren. Die Frage der Qualität der Online-Unterrichte wurde auch unterschiedlich beantwortet. 22 Prozent der Befragten waren mit der Qualität der Unterrichte unzufrieden, während 6 Prozent zu diesem Punkt überhaupt keine Antwort gegeben haben. 72 Prozent der Befragten waren insgesamt mit der Qualität der Unterrichte zufrieden, 28 Prozent davon fanden es nur befriedigend. Auf die dritten und vierten Fragen haben die Studierenden folgendermaßen geantwortet. 12 Teilnehmer sind der Meinung, dass die Aufgaben für Online-Unterrichte gar nicht viel, sondern zu wenig sind. 24 Teilnehmer oder $37 \%$ der befragten fanden den Umfang der Aufgaben als durchschnittlich, während die anderen 28 Teilnehmer die Aufgaben als zu viel bezeichneten.

Die letzten drei Fragen in diesem Teil beziehen sich auf die Fach und IT-Kompetenz der Lehrenden. Die große Mehrheit der Befragten gaben an, dass die Lehrenden mit vereinzelten Ausnahmen ihr Online-Unterricht im Computer durchführten. 4 Testpersonen sind der Meinung, dass die Lehrenden ihr Unterricht nicht gut gehalten haben. Andere 16 Probanden 
fanden den Unterricht der Lehrer eher mittelmäßig, während alle anderen fanden die Qualität der gehaltenen Unterrichte gut bis sehr gut. Fast 19 Prozent der Befragten fanden die Lehrenden in modernen Informationstechnologien nicht kompetent, während weitere 11 Prozent zu dieser Frage keine Antwort gegeben haben. Weitere 18 Prozent sind der Meinung, dass die Lehrenden nur mäßigen Fertigkeiten in modernen Informationstechnologien haben, während andere 52 Prozent der Befragten die IT-Kompetenz der Lehrenden überhaupt nicht bezweifeln.

Die 3 Fragen in der Mitte diesen Teil sind der Fragen des Zeitmanagements, autonomes und selbststeuertes Lernen gewidmet. 21 Testpersonen gaben an, dass sie ihre Zeit gar nicht richtig und effektiv einteilen konnten. Weitere 20 Personen haben das mit Mühe geschaffen, während die anderen 18 Probanden mit dem Zeitmanagement kein Problem hatten. 5 Personen haben dazu keine Angabe gemacht. 17 Studenten wissen überhaupt nicht was autonomes/selbstständiges Lernen ist. Weitere 16 Personen verfügen über ausreichende Kenntnisse zum Thema autonomes/selbstständiges Lernen, während die anderen Teilnehmer mit den Begriffen kein Problem haben. Auf die Frage ob sie die Hausaufgaben selbstständig und autonom machen konnten, haben 6 Testpersonen keine Antwort gegeben. Während 14 Personen mit den Aufgaben zu überfordert fühlten, konnten andere 44 Probanden die Aufgaben selbstständig und autonom meistern, wobei 23 Personen dies mit mäßiger Erfolg gelungen war.

Lernpsychologische Probleme. Beim Online- bzw. Distanzunterricht wird der Lernende öfters selbst entscheiden müssen über den Umfang von Lernstoff, über den Lernort, Lernzeit und den Lerntempo. Und all das verlangt vom Lernenden ein hohes Maß an Selbststeuerung und autonomes Lernen (Vgl. 6, 196). Selbststeuertes und autonomes Lernen sind also die Fertigkeiten, die explizit oder implizit gelernt werden müssen. Wir gehen davon aus, dass die meisten Lernenden diese Fertigkeiten explizit nicht gelernt hatten. Ob die Lernenden sich diese Fertigkeiten implizit gelernt hatten, ist unser Anhaltspunkt. An dieser Stelle kommen die sogenannten Lernstile bzw. Lernerbiographien ins Spiel und es wird ein kleiner Exkurs zum Thema gemacht.

Obwohl die angelsächsische Fremdsprachenerwerbsforschung sehr weit angelegt ist, wird in den meisten einschlägigen Studien die soziale und kulturelle Komponente des Lernens vernachlässigt, da sich in diesen Studien methodologisch nur auf kognitive Komponenten konzentriert werden und die sozial-interaktive Dimension des Lernprozesses häufig außer Acht gelassen werden (Vgl. 3, 854). Neuere Studien bzw. Integrative Ansätze heben den Gegensatz zwischen kognitivistischen Auffassung des Spracherwerbs und auf die kontextuelle Faktoren fokussierende Auffassung auf (Vgl. 11, 886). Ferner in der deutschsprachigen Raum, insbesondere im DaF Kontext findet integrative Forschung von interaktionistischen und soziokulturellen Ansätzen statt (Vgl. 7, 744) und einige deutsche Wissenschaftler sprechen unter dem Themenspektrum Lernervariablen unmittelbar von „Lernertraditionen- bzw. von ,exogenen Faktoren (Vgl. 10; 9). Sie alle gehen allerdings auf die Thematik nicht eingehend ein bzw. behandeln die Thematik unter einem anderen Spektrum (Vgl. 11).

Bisher wurde das Thema der kulturbedingten Lernerbiographien in der usbekischen Fremdsprachenlehrforschung nicht explizit behandelt. Das Thema spielt jedoch eine wichtige Rolle bei der Erstellung der geeigneten einschlägigen Lehrwerke und damit bei der Erzielung größeren Lernerfolge. In der deutschen Fremdsprachenerwerbsforschung befassen sich die 
Wissenschaftler zwar mit der Materie, liegen aber ihren Schwerpunkt auf anderen Punkt, nämlich unter dem Begriff lernexternen Faktoren auf den informellen Erwerb bzw. umgesteuertes Fremdsprachenerwerb im Migrationskontext (Vgl. 11, 886-894). In diesen und anderen Studien wurden zahlreiche Modelle auf der Makro (z.B.: Akkulturationsmodel oder Heidelberger Projekt Pidgin-Deutsch (HPD)) und Mikroebene (z.B.: Mannheimer Projekt) entworfen, in denen einzelne mögliche Faktoren identifiziert, kategorisiert und in Beziehung zueinander gesetzt wurden In all diesen Studien werden allerdings das Zweitsprachenerwerb von Migranten in der informellen Lernkontext untersucht (Vgl. 11, 887-892). Modelle und Studien explizit zur formellen und gesteuerten Fremdsprachenerwerb (Vgl. 7, 738) unter der Berücksichtigung von traditionsbedingten Lernerbiographien wurden bisher nach Auffassung des Autors noch nicht erstellt bzw. (noch) nicht durchgeführt.

Auch wenn auf die methodologische Geschichte des Fremdsprachenlernens zurückgeschaut wird, kann gesehen werden, dass im Gegensatz zu dogmatisch ausgerichteten audio-lingualen und der audiovisuellen Methoden rückte Mitte der 70er Jahre mit dem kommunikativen Ansatz der Lerner in den Mittelpunkt des Lehr und Lernprozesses im Fremdsprachenunterricht. In den 80er und 90er Jahren wurde dann die Lernerorientierung Richtung Individualisierung ausdifferenziert unter dem Stichwort Lehrerautonomie (Vgl. 5, 350). Generell unterscheidet man bei den Lernstilen zwischen Matching und Stretching. Beim Lernstil Matching - Der Lehrende stellt sich auf die Lernpräferenzen der Lernenden ein. Lernaufgabenanpassung, alternative Aufgabenstellung sind typische Merkmale solchen Stils. Voraussetzung für die Lehrenden ist sich Bewusstheit ihres Lernstils. Beim Stretching dagegen die Lernenden passen sich auf die Lehrenden oder den Lernstil an. Im Idealfall die Lehrenden sollen auf die Lernenden orientieren und langsam den Lernenden ermöglichen ihren Lernstil flexibler zu gestalten und so ihren Komfortzonen auszudehnen, denn wenn der Unterrichtsstil auch dem Lernstil des Lernenden entspricht, ist das ein großer Vorteil für Lernenden. Wenn das aber nicht der Fall ist, kann zu Lernschwierigkeiten und Lernstilkonflikten (style war) kommen. Auch die neueren Studien sprechen für lernerorientierter Unterrichtsmethodologien, da ein Lernstil letztendlich zum Bestandteil der Persönlichkeit ist und ein gezielter Einfluss der Persönlichkeit normativ umstritten ist (Vgl. 3, 856-857). Beispielweise Lerner aus buddhistischen Kulturen und anderen kollektiven Gesellschaften wie muslimische Länder sind traditionell gewohnt an lehrerzentrierte Unterrichtsform Daher heißt es „Kulturspezifische und individuelle Traditionen, die sich eher an Regeln am systematischen Vorgehen, am Ausprobieren, an der Autorität des Lehrers und so weiter orientieren, sind daher stärker als bisher auch im Sprachunterricht zu berücksichtigen (Vgl. $10,35)$.

Aus oben genannten Gründen ist es wohl sinnvoll bei der Erstellung von Lehrwerken jeweiligen Lernerbiographien $\mathrm{zu}$ berücksichtigen. Besonderes Augenmerk liegt dabei auf den sogenannte ,(...) exogene Faktoren des Lernumfelds und umfassen damit kollektive und individuelle Aspekte der Umgebung. Dazu gehören kulturelle, moralische, rechtliche, religiöse und andere Werte, Normen und Gewohnheiten, die das Umfeld einer Person bestimmen (Vgl. 10, 32). Und Rohmann (2010) zählt zu den lernerexternen Faktoren die soziale und institutionelle Umgebungsfaktoren, Unterrichts- und Lernsituation, Lehrer und Lehrerverhalten, Lehr und Lernmaterialien, Vermittlungsmethoden sowie Lerngruppe (Vgl. 11, 886). 
Denn, so Roche (2005) und Hufeisen \& Riemer (2010), exogene und endogene Faktoren (Einstellungen, Erfahrungen, Präferenzen, Risikobereitschaft, Toleranzfähigkeit) bzw. personale und soziale Variablen bedingen sich gegenseitig und somit üben Einfluss auf die individuelle Wahrnehmung und Verhalten eines Lerners ein. Die Hauptfrage der vorliegenden Artikel lautet, ob bei der Erstellung von DaF Lehrwerken aus Lernerbiographien ausgehende Lernerbilder berücksichtigt werden sollen bzw. müssen. Die Lernende kommen ja in den Fremdsprachenunterricht nicht wie vom Himmel gefallen, sondern sie bringen ihren Lernertyp, ihren Lernstil, ihre Lernstrategien und Lernerfahrungen in den Lernprozess mit (Vgl. 3, 850; 10, 32), obwohl diese Dispositionen und Gewohnheiten an bestimmte Situationen und Aufgaben nicht gebunden und den Lernenden meistens nicht bewusst sind (5, 349). Das soziale Umfeld, in dem die Menschen aufwachsen prägt das Lernverhalten jedes Einzelnen über Fachgrenzen hinaus und dieses Lebensumfeld aktiviert bzw. entwickelt bei jedem Menschen bestimmte Anlagen und Möglichkeiten weiter (Vgl. 10, 32-40). Und laut Roche ,(j)e früher dies geschieht, desto früher werden die Weichen für ein nachhaltiges und anhaltendes Lernen gestellt" $(10,32)$.

Und wenn wir die Lernerbiographie unserer Lernenden analysieren, so können wir feststellen, dass sie Deutsch nie autonom oder selbstständig gelernt hatten. Sie hatten immer ein Lehrender bei sich entweder in der Schule die gute Deutschlehrerin oder außerhalb der Schule die Nachhilfelehrerin im Fach Deutsch. Und gerade das Fehlen von diesen Fertigkeiten bei den Lernenden überfordert die Lernenden lernpsychologisch und führt dazu, dass sie leicht die Übersicht verlieren und sie in den Online-Unterrichten stets abwesend und diffus einwirken, obwohl sie im Unterricht anwesend sind.

Zusammenfassend kann gesagt werden, dass Online- bzw. Distanzlernen im DaF-Unterricht nicht nur Probleme aufbereitet, sondern wie jedes anderes Phänomen auch gewisse Vorteile hat. Wenn früher die meisten Lernenden und die Lehrenden nur vage Vorstellungen über Onlinebzw. Distanzlernen hatten, haben sie nun diesbezüglich ganz klare und sogar differenzierte Vorstellungen. Corona bedingt entstandene Ad hoc Lösung, könnte in sehr nahen Zukunft den Bildungssektor prägen. Als größtes Problem von Online- bzw. Distanzlernen ist und bleiben die Qualität und Geschwindigkeit der Internetverbindungen und die hohen Preise für Internetzugang. Obwohl seitens der usbekischen Regierung und zuständigen Behörden diesbezüglich enorme Arbeit geleistet und Fortschritte zu verzeichnen sind, sowie nahezu alle Mobilfunkanbieter spezielle günstige Tarife für Studenten anbieten, ist die Lösung dieser Probleme zeit- und institutionell bedingt.

\section{Literaturverzeichnis}

1. Bildungsgesetz der Republik Usbekistan № 637 vom 23.09.2020 Ўзбекистон Республикасининг 2020 йил 23 сентябрдаги №637-сонли “Таълим тўғрисид”ги Қонуни

2. Über die Bestätigung der Studienordnungen in den Fern- und Abendstudiengängen in den Hochschulen - Verordnung des Ministerkabinetts der Republik Usbekistan №930 vom 21.11.2017 Ўзбекистон Республикаси Вазирлар Махкамасининг 2017 йил 21 ноябрдаги №930-сонли „Олий таълим муассасасида сиртқи (махсус сиртқи) ва кечки (сменали) таълимни ташкил этиш тартиби тўғрисидаги низомларни тасдиқлаш хақида“ги Қарори 
3. Aguado, Karin / Riemer, Claudia (2010): Lernstile und Lern(er)typen, in „Deutsch als Fremd- und Zweitsprache. Ein internationales Handbuch“, Hrsg. von Hans-Jürgen Krumm, Christian Fandrych, Britta Hufeisen, Claudia Riemer", 1. Halbband, De Gruyter Moution Verlag, Berlin, S. 850858 ;

4. Die Studienformen: Vollzeit, Teilzeit, Dual, Fernstudium. In: https://www.das-richtige-studieren.de/vor-dem-studium/studienformen/ Zugriff. 12.11.2020

5. Düwell, Henning (2007): Fremdsprachenlerner, in „Handbuch Fremdsprachenunterricht", Hrsg. von Karl Richard Bausch, Herbert Christ, Hans-Jürgen Krumm, 5. Auflage, A.Francke Verlag, Tübingen, S. 347-352; 6. Gutzat, Bärbel (2003): Fremdsprachenlernen über Fernstudien, in „Handbuch Fremdsprachenunterricht", Hrsg. von Karl Richard Bausch, Herbert Christ, Hans-Jürgen Krumm, 4. Auflage, A.Francke Verlag, Tübingen, S. 195-197;

7. Hufeisen, Britta / Riemer, Claudia (2010): Spracherwerb und Sprachenlernen, in „Deutsch als Fremd- und Zweitsprache. Ein internationales Handbuch", Hrsg. von Hans-Jürgen Krumm, Christian Fandrych, Britta Hufeisen, Claudia Riemer“, 1. Halbband, De Gruyter Moution Verlag, Berlin, S. 738-753;

8. Platten, Eva Schaffhausen (2010): Distanz- und Präsenzlernen, in „Deutsch als Fremd- und Zweitsprache. Ein internationales Handbuch“, Hrsg. von Hans-Jürgen Krumm, Christian Fandrych, Britta Hufeisen, Claudia Riemer", 2. Halbband, De Gruyter Moution Verlag, Berlin, S. 11921198 ;

9. Riemer, Claudia (1997): Individuelle Unterschiede im Fremdsprachenerwerb. Die Wechselwirksamkeit ausgewählter Einflussfaktoren, Schneider-Verl. Hohengehren, Baltmannsweiler, 321Seiten;

10. Roche, Jörg (2005): Fremdsprachenerwerb Fremdsprachendidaktik, A Francke Verlag, Tübingen, 282-Seiten;

11. Rohmann, Heike (2010): Lernexterne Faktoren, in „Deutsch als Fremdund Zweitsprache. Ein internationales Handbuch“, Hrsg. von Hans-Jürgen Krumm, Christian Fandrych, Britta Hufeisen, Claudia Riemer", 1. Halbband, De Gruyter Moution Verlag, Berlin, S. 886-894; 
ОНЛАЙН ВА ДИСТАНЩИОН ТАЪЛИМ БЎЙИЧА САВОЛНОМА

(5 баллик бахуолаи икаласида бахуоланг: 1-умуман тўври/тегиили эмас, жуда ёмон, 5 -жуда тугхри/тўлиқ тегишли, аъло)

\section{Умумий маълумотлар}

1.1 Сиз қайси вилоятда истиқомат қиласиз? ㄷрхондарё $\square$ Бошқа вилоят

1.2 Сиз қаерда истиқомат қиласиз? $\square$ Вилоят/туман маркази $\square$ Қишшоқ жойда

1.3 Қайси босқичда? $\quad \square$ 2-босқич $\square$ 3-босқич $\square$ 4-босқич

1.4 Жинсингиз? $\quad \square$ Эркак $\square$ Аёл

1.5 Ёшингиз? $\quad \square 20$ ёшгача $\square 20-25 \square 25-30 \square 30$ ва юқори

1.6 Сиз онлайн-дистанцион таълимни умумий қандай бахолайсиз $\quad$ ㅁ 1 ฉ 3 口 45

1.6 Мен онлайн/дистацион таълимда тўлиқ қатнашдим $\quad \square 1 \square 2 \square 3 \square 4 \square 5$

1.7 Агар сиз онлайн таълимда қатнашмаган бўлсангиз, қайси сабабга кўра қатнашмадингиз (бир қанча сабабларни кўрсатишшнгиз мумкин)

$\square$ Интернет муаммоси (тезлик) $\quad \square$ Телефон муаммоси $\quad \square$ Молиявий муаммо

$\square$ Мотивация/ қизиқиш/ хохиш муаммоси $\square$ Компютер йўқлиги муаммоси

口 Онлайн/дистанцион таълим сифати $\quad \square$ Уй ишлари ва вақт муаммоси

$\checkmark$ Назорат ва тегишли чора йўқлиги $\quad \square$ Интернетдан фойдаланиш муаммоси

Техник муаммолар

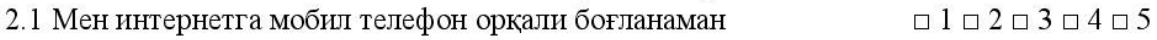

2.2 Мен интернетга ADSL/оптик тола орқали боғланаман $\quad \square 1 \square 2 \square 3 \square 4 \square 5$

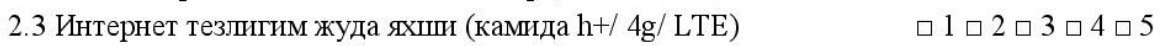

Молиявий масалалар

3.1 Интернет пакетлари қиммат

$\square 1 \square 2 \square 3 \square 4 \square 5$

3.2 Менда талаб даражасидаги компютер бор

3.3 Менда материалларни чиқариш ун принтер бор

$\square 1 \square 2 \square 3 \square 4 \square 5$

3. 4 Менинг замонавий шахсий телефоним бор

$\square 1 \square 2 \square 3 \square 4 \square 5$

3.5 Мен онлайн дарс ун оила азоларимни телефонидан фойдаландим $1 \square 2 \square 3 \square 4 \square 5$

\section{Методик-дидактик муаммолар}

4.1 Фанлар бўйича онлайн ўқув материаллари (дарслик, қўлланма тарқатма материаллар) талаб даражасида

$\square 1 \square 2 \square 3 \square 4 \square 5$

4.2 Фанлар бўйича онлайн дарслар сифати жуда яхши

$\square 1 \square 2 \square 3 \square 4 \square 5$

4.3 Онлайн дарслар бўйича топшириқлар жуда кўп

$\square 1 \square 2 \square 3 \square 4 \square 5$

4.4 Онлайн дарслар буйича топшириқпар жуда кам

$\square 1 \square 2 \square 3 \square 4 \square 5$

4.5 Онлайн дарс топшшриқларини бажаришда вақтимни тўғри ва самарали тақсимлай олдим

$\square 1 \square 2 \square 3 \square 4 \square 5$

4.6 Мен автоном/мустақил ўқиш нималигини биламан

$\square 1 \square 2 \square 3 \square 4 \square 5$

4.7 Мен топшириқларни самарали мустақил/автоном бажара олдим $\square 1 \square 2 \square 3 \square 4 \square 5$

4.8 Фан ўқитувчнси онлайн дарсларни талаб даражасида ўтди $\quad \square 1 \square 2 \square 3 \square 4 \square 5$

4.9 Ўқитувчи замонавий АКТдан фойдаланиш бўйича компетент $\quad \square 1 \square 2 \square 3 \square 4 \square 5$

4.10 Ўқитувчи онлайн-дистанцион дарсни қандай ўтди $\square$ Телефонда $\square$ Компютерда

\section{Шахсга боғлиқ муаммолар}

5.1 Мен барча дарсларда қатнашдим

$\square 1 \square 2 \square 3 \square 4 \square 5$

5.2 Мен фақат мутахассислик фанларида қатнашдим

$\square 1 \square 2 \square 3 \square 4 \square 5$

5.3 Мен фақат НОмутахассислик фанларида қатнашдим

$\square 1 \square 2 \square 3 \square 4 \square 5$

5.4 Онлайн-дарсларда ишттрок қаттиқ назорат қилинди

$\square 1 \square 2 \square 3 \square 4 \square 5$

5.5 Онлайн-дарсларни бахога алоқаси йўқ эди

$\square 1 \square 2 \square 3 \square 4 \square 5$

5.6 Пандемия сабабли ўқитувчилар бахоларни шундай қуйиб берди $\square 1 \square 2 \square 3 \square 4 \square 5$ 


\author{
UMFRAGEBOGEN ZUM THEMA ONLINE BZW. DISTANZLERNEN \\ (Bewerten Sie in 5-Punkte System: 1-trifft gar nicht zu, sehr schlecht \\ 5 -trifft voll zu, ausgezeichnet)
}

Allgemeine Informationen

1.1 In welcher Provinz wohnen Sie? $\square$ In Surchandarja $\square$ In anderem Provinz

1.2 Wo wohnen Sie? $\square$ Im Provinz/Bezirk Zentrum $\square$ Im ländlichen Ort

1.3 In welchem Studienjahr?

$\square 2$. Kurs $\square 3$. Kurs $\square$ 4. Kurs

1.4 Geschlecht?

$\square$ männlich $\square$ weiblich

1.5 Alter $\square$ bis $20 \quad \square 20-25 \square 25-30 \quad \square$ über 30

1.6 Wie bewerten Sie generell Online/Distanzlernen

$\square 1 \square 2 \square 3 \square 4 \square 5$

1.6 Ich nahm in Online/Distanzlernen vollständig teil

$\square 1 \square 2 \square 3 \square 4 \square 5$

1.7 Falls nein, aus welchem Grund konnten Sie in Online/Distanzlernen nicht teilnehmen (mehrere Antworten möglich)

$\square$ Internetzugang (Geschwindigkeit) $\quad \square$ Handyproblem $\quad \square$ Finanzielle Probleme

$\square$ Motivation/ Interesse/ Wille

$\square$ Qualität des Online/Distanzunterrichts $\quad \square$ Zeitmangel wegen Hausangelegenheiten

$\square$ Fehlende Kontrolle der Anwesenheit u Hausaufgaben $\square$ Kenne mich im Internet nicht gut aus

Technische Probleme

2.1 Ich habe Zugang zum Internet via mein mobil Handy

2.2 Ich habe Festnetz/ADSL-Anschluss zum Internet

$\square 1 \square 2 \square 3 \square 4 \square 5$

$\square 1 \square 2 \square 3 \square 4 \square 5$

2.3 Meine Internetgeschwindigkeit ist sehr gut $(\mathrm{h}+/ 4 \mathrm{~g} / \mathrm{LTE})$

$\square 1 \square 2 \square 3 \square 4 \square 5$

3.1 Internetpakete sind teuer

Finanzielle Probleme

3.2 Ich habe einen modernen PC

$\square 1 \square 2 \square 3 \square 4 \square 5$

3.3 Ich habe ein Drucker zum Drucken von Materialien

$\square 1 \square 2 \square 3 \square 4 \square 5$

3.4 Ich habe modernes, eigenes Handy

$\square 1 \square 2 \square 3 \square 4 \square 5$

3.5 Ich habe für das Online-Unterricht Handy von meinen Eltern benutzt $\square 1 \square 2 \square 3 \square 4 \square 5$

Methodisch-didaktische Probleme

4.1 Online-Lehrmaterialien (Lehrbuch, Materialien, Handouts) sind in guter Qualität

4.2 Qualität der Online-Unterrichte sind sehr gut

$\square 1 \square 2 \square 3 \square 4 \square 5$

4.3 Die Aufgaben für Online-Unterricht sind zu viel

$\square 1 \square 2 \square 3 \square 4 \square 5$

$\square 1 \square 2 \square 3 \square 4 \square 5$

4.4 Die Aufgaben für Online-Unterricht sind zu wenig

$\square 1 \square 2 \square 3 \square 4 \square 5$

4.5 Ich konnte bei der Erfüllung der Hausaufgaben für Online-

Unterricht meine Zeit richtig und effektiv einteilen

$\square 1 \square 2 \square 3 \square 4 \square 5$

4.6 Ich weiß was Autonomes/Selbstständiges Lernen ist

$\square 1 \square 2 \square 3 \square 4 \square 5$

4.7 Ich konnte Aufgaben autonom/selbstständig machen

$\square 1 \square 2 \square 3 \square 4 \square 5$

$\square 1 \square 2 \square 3 \square 4 \square 5$

4.9 Der Lehrer ist in modernen Informationstechnologien kompetent

$\square 1 \square 2 \square 3 \square 4 \square 5$

4.10 Der Lehrer unterrichtete den Online-Unterricht

$\square$ mit Handy $\square$ mit PC

\title{
Persönliche Probleme
}

5.1 Ich habe an Online-Unterrichten in allen Fächern teilgenommen $\quad \square 1 \square 2 \square 3 \square 4 \square 5$

5.2 Ich habe an Online-Unterricht NUR im Hauptfächern teilgenommen $\square 1 \square 2 \square 3 \square 4 \square 5$

5.3 Habe an Online-Unterrichten NUR in Nebenfächern teilgenommen $\quad \square 1 \square 2 \square 3 \square 4 \square 5$

5.4 Die Teilnahme an Online-Unterrichten wurden streng kontrolliert $\quad \square 1 \square 2 \square 3 \square 4 \square 5$

5.5 Online-Unterrichte hatten keinen direkten Bezug zu Note $\quad \square 1 \square 2 \square 3 \square 4 \square 5$

5.6 Wegen der Pandemie gaben die Lehrer die Noten einfach so $\quad \square 1 \square 2 \square 3 \square 4 \square 5$ 